\title{
Effects of fluoxetine on brain-derived neurotrophic factor serum concentration and cognition in patients with vascular dementia
}

This article was published in the following Dove Press journal:

Clinical Interventions in Aging

10 March 2014

Number of times this article has been viewed

\author{
Xuan Liu', \\ Junjian Zhang' \\ Dong Sun' \\ Yuanteng Fan' \\ Hongbin Zhou ${ }^{2}$ \\ Binfang $\mathrm{Fu}^{2}$ \\ 'Department of Neurology, Zhongnan \\ Hospital, Wuhan University, Wuhan, \\ ${ }^{2}$ Department of Neurology, Xiangyang \\ Central Hospital, Medical College, \\ Hubei University of Arts and Science, \\ Xiangyang, Hubei, People's Republic \\ of China
}

\begin{abstract}
Background: Selective serotonin reuptake inhibitors improve cognition in patients with stroke and increase the expression of brain-derived neurotrophic factor (BDNF) in the rat hippocampus. However, the effects of selective serotonin reuptake inhibitors on cognition and serum BDNF levels in patients with vascular dementia are largely unknown. We performed an open-label study to investigate the effects of fluoxetine, a selective serotonin reuptake inhibitor, on cognition and serum BDNF levels in patients with vascular dementia.
\end{abstract}

Methods: Fifty patients with vascular dementia were randomly allocated to receive fluoxetine ( $20 \mathrm{mg} /$ day; $\mathrm{n}=25$ ) or no fluoxetine (control group; $\mathrm{n}=25$ ) for 12 weeks. Both groups received secondary prevention of stroke. Serum BDNF level, Mini-Mental State Examination (MMSE) score, Ten-Point Clock Drawing score, and Digit Span Test and Verbal Fluency Test scores were measured at baseline and at week 12 in the both groups.

Results: The baseline serum BDNF level correlated significantly with the MMSE score. MMSE score, Ten-Point Clock Drawing score, and serum BDNF level increased significantly in the fluoxetine group but not in the control group. The increase in serum BDNF level correlated with the increase in MMSE score in the fluoxetine group.

Conclusion: Fluoxetine may potentially improve cognition in patients with vascular dementia and requires further investigation. BDNF may play an important role in cognitive recovery.

Keywords: brain-derived neurotrophic factor, cognition, fluoxetine, improvement, neuroplasticity, vascular dementia

\section{Introduction}

Vascular dementia is a severe stage of vascular cognitive impairment, which is a spectrum of cognitive impairments caused by or associated with vascular factors. ${ }^{1}$ In the People's Republic of China, up to $30 \%$ of stroke patients develop post-stroke cognitive impairment or delayed dementia after stroke. ${ }^{2}$ However, no drugs are licensed for the treatment of vascular cognitive impairment, and treatment is limited to controlling known vascular risk factors. Recently, selective serotonin reuptake inhibitors (SSRIs), which are antidepressants, have been shown to improve motor function and cognition in patients with stroke. ${ }^{3,4}$ A small retrospective clinical study of selective serotonin reuptake inhibitors showed benefits on the Executive Interview (EXIT-25), an executive function test, in patients with vascular cognitive impairment. ${ }^{5} \mathrm{~A}$ preclinical study found that antidepressants including SSRIs had direct effects on brain function associated with plasticity, neurogenesis, and neuronal differentiation. ${ }^{6}$

Brain-derived neurotrophic factor (BDNF) is an important member of the neurotrophin family of growth factors. BDNF is abundant in the brain and periphery, and plays
Department of Neurology, Zhongna Hospital, Wuhan University, I69 Donghu Road, Wuhan 43007I, People's Republic of China

Tel +86 27678 I 2885

Fax +86 2768758670

Emailwdsjkx@I63.com 
a key role in regulating survival, growth, and maintenance of neurons. ${ }^{7,8}$ In addition to its involvement in neuronal survival, growth, and differentiation, BDNF plays a crucial role in learning and memory. ${ }^{9}$ A recent study showed that older adult heterozygous BDNF knockout mice, which have a chronic $50 \%$ lack of BDNF, are deficient for extinction memory. ${ }^{10}$ BDNF has been shown to play a large role in reducing neuroplasticity in aged rats. ${ }^{11}$ Numerous clinical studies have found that chronic use of antidepressants increases serum BDNF levels in patients with depression. ${ }^{12-16}$ Further, studies have also shown that chronic administration of antidepressants increases the expression of cyclic adenosine monophosphate response element binding protein, an important upstream signaling molecule of BDNF, and neurogenesis in the adult rat hippocampus. ${ }^{17,18}$ In light of the observed beneficial effects of antidepressants, we hypothesized that SSRIs would improve cognitive function and increase serum BDNF levels in patients with vascular dementia.

\section{Patients and methods Study design and patients}

This prospective, open-label study was designed to detect a clinically meaningful difference in cognition between two groups. Assuming a within-group standard deviation of 2.0 for the Mini-Mental State Examination (MMSE) test and 1.0 for the Ten-Point Clock (TPC) drawing test, we calculated that a sample size of 50 (minimum of 25 treated subjects and 25 controls) would be needed to detect a one-point difference in mean change from baseline on the MMSE test and a 0.5-point difference on the TPC test with a statistical power of $80 \%$ at a $5 \%$ significance level according to the results of previous trials. Patients with vascular dementia were recruited from Zhongnan Hospital at Wuhan University and Xiangyang Central Hospital from June 2012 to June 2013.

The inclusion criteria were: age 45-80 years; history of stroke and/or neuroimaging (computed tomography or magnetic resonance imaging) showing evidence of cerebrovascular disease; cognitive impairment meeting the Diagnostic and Statistical Manual of Mental Disorders Fourth Edition (DSM-IV) criteria for dementia; ${ }^{19}$ cognitive impairment deemed to have a vascular cause (by evidence of sudden onset, stepwise progression, patchy cortical deficits on cognitive testing, other evidence of atherosclerosis, focal neurological findings, and neuroimaging data); and a Hachinski Ischemic Score of more than $7 .{ }^{20}$

Participants were excluded if they met the DSM-IV diagnostic criteria for depressive disorder. ${ }^{19}$ Other exclusion criteria included: heart failure, respiratory failure, other organ failure, pregnancy, or a tumor that could put the patient at special risk; a history of neurodegenerative diseases including Parkinson's disease, Alzheimer's disease, multiple system atrophy, and Huntington's disease that could interfere with the efficacy evaluations; inability to perform neuropsychological tests due to severe aphasia; history of alcohol and/or drug abuse; and use of anticholinesterase, anticoagulant, or other drugs that could potentially improve cognition within one year prior to enrollment.

All patients underwent a standardized baseline assessment including a physical and neurological examination. In addition, computed tomography or magnetic resonance imaging was performed to validate the diagnosis of vascular dementia. Lues serology (a serologic test for syphilis), vitamin B12, folic acid, and thyroxine levels were examined to exclude other causes of dementia. Patients were interviewed to ascertain whether they met the DSM-IV diagnostic criteria for depression ${ }^{19}$ at the 12-week follow-up and, if so, they were excluded.

Allocation of patients to the fluoxetine group or the control group was determined by reference to a statistical series based on a random number table drawn up for each patient at each center by one of the investigators (DS). The investigators were aware of the allocation of participants but the data collectors were not. Data analysis was done by the first author using a deidentified database. Patients with vascular dementia in the fluoxetine group received fluoxetine $20 \mathrm{mg} /$ day and secondary prevention of stroke for 12 weeks, whereas the control group received only secondary prevention of stroke for 12 weeks. Secondary prevention of stroke involves controlling known vascular risk factors. Patients were followed up at weeks 4 and 8 by telephone and at week 12 by a visit to the clinic. Information regarding any adverse reactions was collected at each follow-up visit.

The primary outcome in both groups was cognitive function as assessed by an experienced neuropsychologist using the $\mathrm{MMSE}^{21}$ and $\mathrm{TPC}^{22}$ at baseline and week 12. The secondary outcomes in both groups were serum BDNF levels and Digit Span Test and Verbal Fluency Test (VFT) scores at baseline and at week 12. The MMSE was used to assess global cognitive function, and executive function was assessed using the TPC. The Digit Span Test ${ }^{23}$ required participants to repeat orally verbally presented series of numbers of increasing length, first forward and then backward. The VFT $^{24}$ evaluates the ability to form and fluently utter words compatible with given criteria. The participants were required to list in one minute as many words as possible that belong 
to a given semantic category (fruit, vegetable, and animal). The score was the sum of three one-minute trials with different letters.

The study was performed according to the Declaration of Helsinki and its subsequent revisions, and was approved by all relevant institutional review boards. Written informed consent was obtained from each participant (or their legal representative).

\section{Measurement of serum BDNF level}

Blood (4 mL) was drawn into serum tubes between 9 am and $11 \mathrm{am}$ and kept for one hour at room temperature followed by one hour at $4{ }^{\circ} \mathrm{C} .{ }^{25}$ Serum samples were then isolated (by centrifugation at $2,000 \times g$ at $4^{\circ} \mathrm{C}$ for 15 minutes) and stored at $-80^{\circ} \mathrm{C}$ until further analysis. BDNF levels were measured by enzyme-linked immunosorbent assay using a commercially available kit (RayBiotech, Inc., Norcross, GA, USA) according to the manufacturer's protocol. Briefly, samples were added to 96-well plates coated with anti-BDNF monoclonal antibody, incubated at $37^{\circ} \mathrm{C}$ for 2 hours, and washed five times with appropriate washing buffer. The plates were incubated with anti-human BDNF polyclonal antibody at room temperature for 2 hours, washed, and incubated with anti-IgY antibody conjugated with horseradish peroxidase for one hour at room temperature. The plates were then incubated in peroxidase substrate and tetramethylbenzidine solution to produce a color reaction. The reaction was terminated with $1.0 \mathrm{M} \mathrm{HCl}$, and the optical density of the color reaction in the wells was measured at $450 \mathrm{~nm}$ using an Emax plate reader to determine BDNF values. All samples and standards were assayed in duplicate. The minimal concentration of BDNF for detection is commonly lower than $80 \mathrm{pg} / \mathrm{mL}$.

\section{Statistical analysis}

The data are presented as the mean \pm standard deviation. Baseline characteristics were compared across groups using a two-samples $t$-test (or the Mann-Whitney $U$ test for data with a non-normal distribution). The sex distribution was compared across the groups using Pearson's $\chi^{2}$ test. Correlations between serum BDNF levels and clinical variables were assessed using bivariate Pearson and Spearman correlation coefficients. Changes in MMSE scores, TPC scores, Digit Span Test scores, VFT scores, and serum BDNF levels were compared between the two groups using the two-samples $t$-test (or the Mann-Whitney $U$ test for data with a nonnormal distribution). The statistical significance of the results was set at $P<0.05$. All statistical analyses were carried out using Statistical Package for the Social Sciences version 12 software (SPSS Inc., Chicago, IL, USA).

\section{Results}

Between June 9, 2012 and June 10, 2013, a total of 50 patients were enrolled in the study. Twenty-five patients were assigned to the fluoxetine group and 25 patients were assigned to the control group. All participants had a history of stroke at least 3 months earlier and attributed to large artery atherosclerosis (14 in the fluoxetine group, 15 in the control group), small artery occlusion (five in the fluoxetine group, six in the control group), cardioembolism (three in the fluoxetine group, two in the control group), or unidentified/other (three in the fluoxetine group, two in the control group). All patients completed their 12-week follow-up (see Flow diagram).

Table 1 shows the demographic and clinical characteristics of patients in the fluoxetine and control groups. We found no statistically significant differences between the two groups with respect to age $(P=0.562)$, sex $(P=0.777)$, body mass index $(P=0.734)$, years of education $(P=0.306)$, smoking $(P=0.771)$, hypertension $(P=0.564)$, diabetes mellitus $(P=0.395)$, hypercholesterolemia $(P=0.571)$, MMSE score $(P=0.490)$, TPC score $(P=0.330)$, Digit Span Test score $(P=0.251)$, VFT score $(P=0.231)$, or serum BDNF level $(P=0.885)$.

Mean baseline and week 12 BDNF levels are shown in Table 2. There was a significant change in mean serum BDNF levels from baseline between the fluoxetine and control groups $(P<0.0001)$. Mean changes in MMSE, TPC, Digit Span Test, and VFT scores are also shown in Table 2. There were significant differences in mean change from baseline between the fluoxetine and control groups in the primary endpoints of MMSE $(P=0.031)$ and TPC $(P=0.007)$ scores, but we found no significant differences in Digit Span Test score $(P=0.342)$ or VFT score $(P=0.660$, Table 2$)$.

The baseline serum BDNF level (across groups) was significantly correlated with baseline MMSE score ( $r=0.326, P=0.021$, Figure 2). However, the baseline serum BDNF level was not significantly correlated with age $(r=-0.132, P=0.360)$, sex $(r=-0.253, P=0.076)$, body mass index $(r=-0.019, P=0.895)$, or years of education ( $r=0.160, P=0.268$ ). Further, in the fluoxetine group, the change in serum BDNF level from baseline to week 12 was significantly correlated with the change in MMSE score ( $r=0.413, P=0.04$, Figure 3 ).

Fluoxetine was well tolerated. The main adverse events were dry mouth (four in the fluoxetine group, three controls) and transient gastrointestinal symptoms, including nausea 


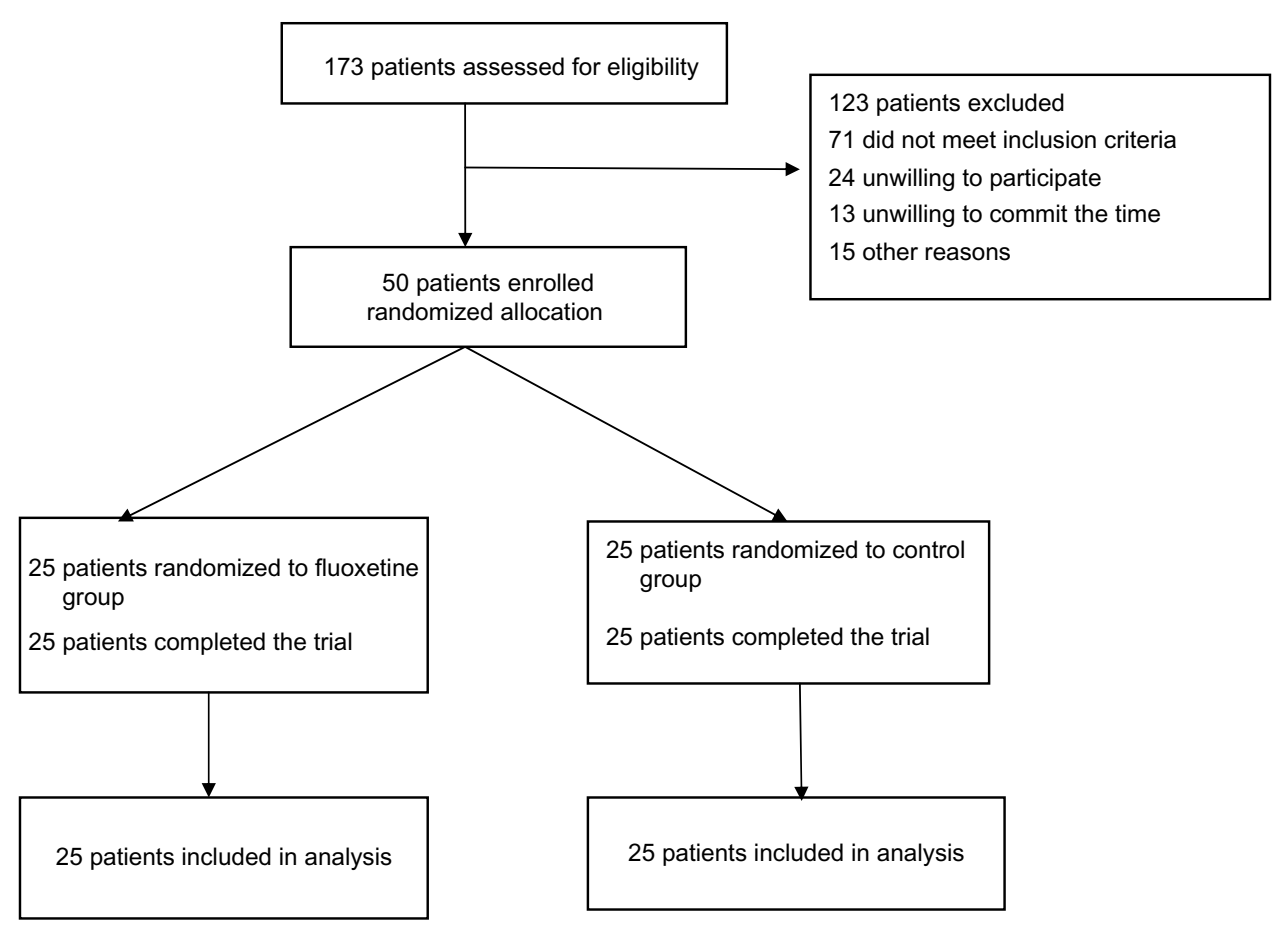

Figure I Flow diagram Trial profile.

(six in the fluoxetine group, seven controls) and diarrhea (three in the fluoxetine group, three controls). No deaths occurred in either group, and no differences between groups in the frequency of any of these events were found. Treatment was not interrupted in any patients with adverse events.

\section{Discussion}

In the present study, we investigated the effects of fluoxetine on cognition and serum BDNF levels in patients with vascular dementia. Compared with patients receiving only secondary prevention of stroke, patients also receiving fluoxetine showed

Table I Demographic and clinical characteristics of patients with vascular dementia randomized to the fluoxetine and control groups

\begin{tabular}{llll}
\hline Characteristic & $\begin{array}{l}\text { Fluoxetine } \\
\text { group }\end{array}$ & $\begin{array}{l}\text { Control } \\
\text { group }\end{array}$ & P-value \\
\hline Sex (male/female), n & $\mathrm{I} 3 / \mathrm{I} 2$ & $\mathrm{I} 4 / \mathrm{II}$ & 0.777 \\
Age (years), mean (SD) & $66.4(7.7)$ & $67.8(9.2)$ & 0.562 \\
BMI (kg/m²), mean (SD) & $23.3(\mathrm{I} .8)$ & $23.5(2.0)$ & 0.734 \\
Education (years), mean (SD) & $8.6(4.5)$ & $9.4(3.8)$ & 0.306 \\
MMSE score, mean (SD) & $\mathrm{I} 5 . \mathrm{I}(3.0)$ & $\mathrm{I} 5.7(2.6)$ & 0.490 \\
TPC score, mean (SD) & $3.7(\mathrm{I} .2)$ & $4.1(\mathrm{I} . \mathrm{I})$ & 0.330 \\
VFT score, mean (SD) & $\mathrm{I} 4.7(4.5)$ & $\mathrm{I} 5.7(4.2)$ & $0.23 \mathrm{I}$ \\
Digit Span score, mean (SD) & $4.6(\mathrm{I} .3)$ & $4.9(\mathrm{I} .2)$ & $0.25 \mathrm{I}$ \\
BDNF (ng/mL), mean (SD) & $2 \mathrm{I} .2(3.2)$ & $2 \mathrm{I} .4(5.8)$ & 0.885 \\
\hline Ab
\end{tabular}

Abbreviations: BDNF, brain-derived neurotrophic factor; BMI, body mass index; MMSE, Mini-Mental State Examination; n, number of patients; SD, standard deviation; TPC, Ten-Point Clock Drawing; VFT, Verbal Fluency Test. higher scores in a neuropsychological test assessing global cognitive function and executive function. Previous studies found that fluoxetine improved cognitive impairment after neonatal hypoxic-ischemic brain injury in rats. ${ }^{26}$ In addition, a clinical study reported that fluoxetine enhanced memory and cognition in patients with mild cognitive impairment, ${ }^{27}$ and sertraline improved executive function in patients with vascular cognitive impairment. ${ }^{5}$ Moreover, citalopram improved dexterity in chronic stroke patients, ${ }^{28}$ and escitalopram was associated with improved cognitive recovery following stroke compared with placebo and problem-solving therapy. ${ }^{4}$

Table 2 Mean change from baseline and week 12 in MMSE score, TPC score, Digit Span Test score, and VFT score, and serum BDNF level in patients with vascular dementia stratified by treatment group

\begin{tabular}{|c|c|c|c|c|c|}
\hline & \multicolumn{2}{|l|}{ Week I2 } & \multicolumn{3}{|c|}{$\begin{array}{l}\text { Mean change from baseline } \\
\text { to week } 12\end{array}$} \\
\hline & $\begin{array}{l}\text { Fluoxetine } \\
\text { mean } \\
\text { (SD) }\end{array}$ & $\begin{array}{l}\text { Control } \\
\text { mean } \\
(\mathrm{SD})\end{array}$ & $\begin{array}{l}\text { Fluoxetine } \\
\text { mean } \\
\text { (SD) }\end{array}$ & $\begin{array}{l}\text { Control } \\
\text { mean } \\
\text { (SD) }\end{array}$ & $P$-value \\
\hline MMSE & I6.I (2.9) & I5.8 (2.7) & $1.0(1.2)$ & $0.2(1.4)$ & 0.031 \\
\hline TPC & $4.5(1.4)$ & $4.3(1.2)$ & $0.8(0.9)$ & $0.2(0.9)$ & 0.007 \\
\hline Digit & $5.0(1.3)$ & $5.1(0.9)$ & $0.4(0.9)$ & $0.2(1.0)$ & 0.342 \\
\hline \multicolumn{6}{|l|}{ Span } \\
\hline VFT & $15.0(5.2)$ & I $6.2(4.1)$ & $0.3(2.2)$ & $0.5(2.1)$ & 0.660 \\
\hline BDNF (ng/ml) & $25.7(4.0)$ & $21.5(6.9)$ & $4.5(3.7)$ & $0.1(3.2)$ & $<0.0001$ \\
\hline
\end{tabular}

Abbreviations: BDNF, brain-derived neurotrophic factor; MMSE, Mini-Mental State Examination; SD, standard deviation; TPC, Ten-Point Clock Drawing; VFT, Verbal Fluency Test. 


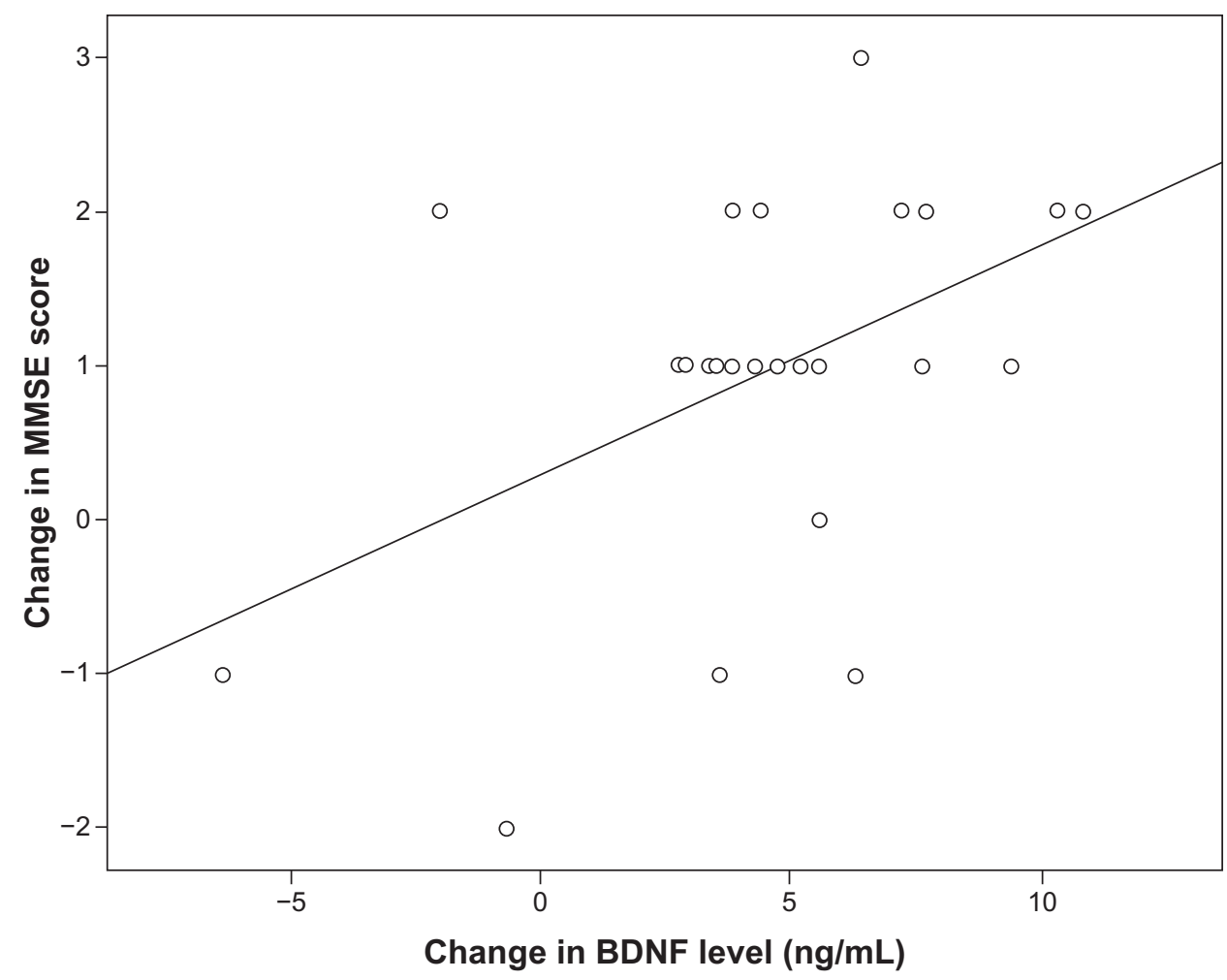

Figure 2 Correlation between baseline serum BDNF level (across groups) and MMSE score $(r=0.326, P=0.021)$.

Abbreviations: BDNnF, brain-derived neurotrophic factor; MMSE, Mini-Mental State Examination.

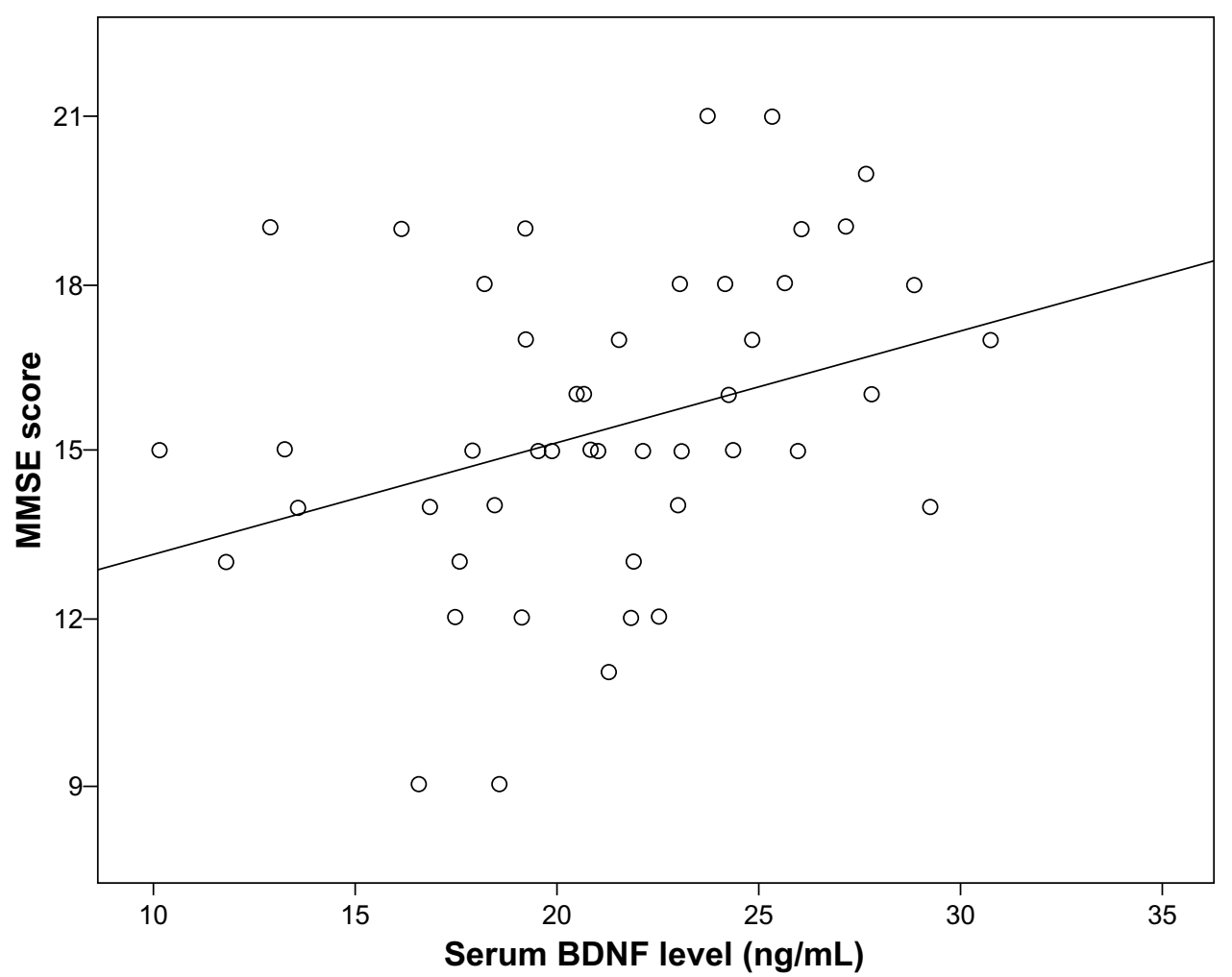

Figure 3 Correlation between change in serum BDNF level and change in MMSE score in the fluoxetine group. Note that "change in MMSE score" is the week 12 score minus the baseline score and that "change in BDNF level" is the week 12 serum BDNF level minus the baseline serum BDNF level ( $r=0.4 \mid 3, P=0.04)$. Abbreviations: BDNF, brain-derived neurotrophic factor; MMSE, Mini-Mental State Examination. 
Our study is consistent with these previous studies. We found improvement in cognitive function, especially executive function, following treatment with fluoxetine in patients with vascular dementia. Thus, SSRIs may be an alternative treatment for improving cognitive impairment in patients with vascular dementia. Growing evidence suggests that serotonin may have a regulatory effect on memory function in healthy individuals, particularly for restoring impaired cognition due to serotonin dysfunction. ${ }^{29}$ For instance, acute tryptophan depletion, which reduces serotonergic neurotransmission, is associated with long-term memory impairment independent of mood or attentional changes. ${ }^{30}$

Cognitive recovery is a complex process that is mediated by multiple mechanisms, including resolution of diaschisis, enhanced regional metabolism, and neuroplastic changes. ${ }^{31}$ Increasing evidence also shows that SSRIs produce neuroplastic changes in the hippocampus and cerebral cortex. For instance, fluoxetine increased the proliferation of neuronal precursors in the subgranular zone of the dentate gyrus ${ }^{6}$ and restored cortical plasticity in the visual system of adult amblyopic rats, fostering the recovery of visual function. ${ }^{32}$ This effect is probably mediated by a decrease in intracortical inhibition and increased expression of BDNF. ${ }^{32}$ Further, SSRIs may have improved motor performance, prevented brain atrophy, and delayed mortality in a rat model of Huntington's disease. ${ }^{33}$ Thus, we speculate that the improvement in global cognitive function observed in our study may be partly related to remodeling of the hippocampal circuitry and increased dentate gyrus neurogenesis. However, a small clinical study did not find improved memory in patients with Alzheimer's disease who were treated with fluoxetine. ${ }^{34}$ The utility of SSRIs for cognitive recovery in patients with vascular dementia should be investigated further in future studies.

We also investigated the effect of fluoxetine on serum BDNF levels in patients with vascular dementia and found that fluoxetine significantly increased the level of serum BDNF. In 2002, Karege et al reported that the serum BDNF level was lower in depressed individuals than in healthy controls, ${ }^{25}$ and antidepressant treatment increases the serum BDNF levels in patients with major depressive disorder. ${ }^{12-16}$ In a post mortem human brain tissue study, an increase in BDNF expression was reported in subjects treated with antidepressant drugs compared with untreated individuals. ${ }^{35}$ Our findings are in line with these previous studies showing increased serum BDNF levels in patients with vascular dementia who are treated with fluoxetine.

The main source of serum BDNF in humans is platelets, ${ }^{36-38}$ and the average serum BDNF level is more than 100-fold higher than the average plasma BDNF level in humans because the main source of serum BDNF is platelets. ${ }^{39}$ This difference is due to degranulation of platelets during the clotting process. ${ }^{40}$ Potential cellular sources of BDNF in plasma are vascular endothelial cells, ${ }^{41}$ smooth muscle cells, ${ }^{42}$ and activated macrophages or lymphocytes. ${ }^{43,44}$ There is evidence showing that BDNF can cross the blood-brain barrier, ${ }^{45,46}$ and a positive correlation was found between serum and cortical BDNF levels. ${ }^{47}$ According to these results, BDNF changes in the central nervous system of patients with vascular dementia may be correlated with changes of BDNF serum levels. However, further studies are needed to examine the exact changes in BDNF in the central nervous system following treatment with SSRIs.

Vascular cognitive impairment is a syndrome with evidence of clinical stroke or subclinical vascular brain injury and cognitive impairment affecting at least one cognitive domain. The most severe form of vascular cognitive impairment is vascular dementia. ${ }^{48}$ Vascular dementia describes a group of vessel disorders with different types of vascular lesions that finally contribute to the development of dementia. ${ }^{48}$ Vascular risk factors are the most important contributors to vascular cognitive impairment and vascular dementia. BDNF and TrK receptors play an important role in development of vascular systems and response to injury. A preclinical study showed that cerebrovascular BDNF protein was reduced in the cortical endothelium in 6-month diabetic rats and that reduction of BDNF occurs early after induction of diabetes. ${ }^{49}$ Moreover, BDNF plays an important role during aging and development of chronic hypertension. ${ }^{50}$ The effects of chronic and acute treatment with SSRIs on tropomyosin-related kinase (TrkB) autophosphorylation and TrkB protein levels have been tested in animal studies. TrkB is a high-affinity receptor for BDNF, and its autophosphorylation results in activation and serves as an indirect signal of neuronal release. ${ }^{51}$ Antidepressants increase TrkB autophosphorylation in the anterior cingulate cortex and hippocampus of mice within 30-60 minutes of a single injection, but no acute treatment with antidepressants influence the expression levels of total TrkB protein. ${ }^{51}$ In a preclinical study, escitalopram increased BDNF and TrkB mRNA levels in the hippocampus and prefrontal cortex of juvenile rats after 4 days of treatment. ${ }^{52}$ Several studies have demonstrated that SSRIs promote neurogenesis in the hippocampus. ${ }^{17,18}$ Further, the evidence also shows that blockade of hippocampal neurogenesis diminishes the antidepressant-like effects of fluoxetine, suggesting involvement of BDNF in antidepressant actions. ${ }^{53}$ In another study, experimentally lowering brain BDNF or lowering expression of TrkB in neural progenitor cells of the hippocampus diminished antidepressant efficacy 
in animal models. ${ }^{54}$ The findings of these previous studies illustrate how BDNF is influenced by a complex cascade of molecular mechanisms mediated by SSRIs. Thus, upregulation of BDNF may mediate at least part of the neuroprotective effect of SSRIs. Given that fluoxetine administration upregulates BDNF, it is intriguing that the effect of fluoxetine in improving cognitive recovery in patients with vascular dementia may be related to this mechanism.

We also found that a higher serum BDNF level was correlated with a better MMSE score in patients with vascular dementia. Gunstad et al found that higher serum BDNF levels were associated with better neuropsychological function in healthy older adults. ${ }^{55}$ In addition, past clinical studies have shown that higher serum BDNF levels are related to better MMSE test performance in patients with early Alzheimer's disease or vascular dementia. ${ }^{56,57}$ Our findings are consistent with these studies, and suggest an important role for BDNF in learning and memory ${ }^{9}$ and in maintaining synaptic plasticity, which is involved in functions such as long-term potentiation. ${ }^{7}$ Our findings are also consistent with findings showing that BDNF is affected at different levels in aged rats with global impairment, including reduced transcription, impaired protein synthesis and processing, and decreased activation of TrkB receptors. ${ }^{11}$ Further, the increase in serum BDNF level was significantly correlated with the increase in MMSE score, and the upregulation of serum BDNF levels was paralleled by cognitive improvement in patients with vascular dementia in our study. Our findings are in line with the neurotrophin hypothesis, which states that an increase in BDNF level is an attempt to reorganize neuronal connectivity, leading to increased neuronal survival and differentiation.? These findings suggest a relationship between expression and secretion of BDNF and improvement in cognition in patients with vascular dementia treated with fluoxetine. BDNF may play an important role in cognitive recovery. Thus, further studies are needed to reach a clear conclusion about the role of BDNF in patients with vascular dementia treated with fluoxetine.

To our knowledge, this is the first study describing the impact of SSRIs on serum BDNF level and MMSE score in patients with vascular dementia. However, we must note that this analysis is limited by the small sample size and openlabel drug administration protocol used. Another limitation of the study is that the patients were followed for only 12 weeks, and maintenance therapy was not included. The benefits observed in our study may have been only transient. Larger double-blind, placebo-controlled studies with a longer period of follow-up are needed to confirm these findings.

\section{Conclusion}

In our study, we found that fluoxetine was associated with improved cognitive recovery and a significant increase in serum BDNF levels in patients with vascular dementia when compared with matched controls who did not receive fluoxetine. The utility of SSRIs in patients with vascular dementia requires further investigation.

\section{Acknowledgment}

This study was supported by funds from the Scientific and Technical Key Project of Hubei Province of the People's Republic of China (2007AA302B09).

\section{Disclosure}

The authors report no conflicts of interest in this work.

\section{References}

1. Rockwood K, Ebly E, Hachinski V, et al. Presence and treatment of vascular risk factors in patients with vascular cognitive impairment. Arch Neurol. 1997;54:33-39.

2. Zhou DH, Wang JY, Li J, et al. Frequency and risk factors of vascular cognitive impairment three months after ischemic stroke in china: the Chongqing Stroke Study. Neuroepidemiology. 2005;24:87-95.

3. François Chollet JT, Jean-François Albucher. Fluoxetine for motor recovery after acute ischaemic stroke (FLAME) a randomised placebocontrolled trial. Lancet Neurol. 2011;10:123-130.

4. Jorge RE, Acion L, Moser D, Adams HP Jr, Robinson RG. Escitalopram and enhancement of cognitive following stroke. Arch Gen Psychiatry. 2010;67:187-196.

5. Donald R, Royall MD. Sertraline improves executive function in patients with vascular cognitive impairment. J Neuropsychiatry Clin Neurosci. 2009;21:445-454.

6. Santarelli L, Saxe M, Gross C, et al. Requirement of hippocampal neurogenesis for the behavioral effects of antidepressants. Science. 2003;301:805-809.

7. Schinder AF, Poo M. The neurotrophin hypothesis for synaptic plasticity. Trends Neurosci. 2000;23:639-645.

8. Pencea V, Bingaman KD, Wiegand SJ, et al. Infusion of brain-derived neurotrophic factor into the lateral ventricle of the adult rat leads to new neurons in the parenchyma of the striatum, septum, thalamus, and hypothalamus. J Neurosci. 2001;21:6706-6717.

9. Yamada K, Nabeshima T. Brain-derived neurotrophic factor/ TrkB signaling in memory processes. J Pharmacol Sci. 2003;91: 267-270.

10. Psotta L, Lessmann V, Endres T. Impaired fear extinction learning in adult heterozygous BDNF knock-out mice. Neurobiol Learn Mem. 2013;103:34-38.

11. Calabrese F, Guidotti G, Racagni G, et al. Reduced neuroplasticity in aged rats: a role for the neurotrophin brain-derived neurotrophic factor. Neurobiol Aging. 2013;34:2768-2776.

12. Matrisciano F, Bonaccorso S, Ricciardi A, et al. Changes in BDNF serum levels in patients with major depression disorder (MDD) after 6 months treatment with sertraline, escitalopram, or venlafaxine. J Psychiatr Res. 2009;43:247-254.

13. Lee HY, Kim YK. Plasma brain-derived neurotrophic factor as a peripheral marker for the action mechanism of antidepressants. Neuropsychobiology. 2008;57:194-199.

14. Shimizu E, Hashimoto K, Okamura N, et al. Alterations of serum levels of brain-derived neurotrophic factor (BDNF) in depressed patients with or without antidepressants. Biol Psychiatry. 2003;54:70-75. 
15. Piccinni A, Marazziti D, Catena M, et al. Plasma and serum brainderived neurotrophic factor (BDNF) in depressed patients during 1 year of antidepressant treatments. J Affect Disord. 2008;105: 279-283.

16. Deuschle M, Gilles M, Scharnholz B, et al. Changes of serum concentrations of brain-derived neurotrophic factor (BDNF) during treatment with venlafaxine and mirtazapine: role of medication and response to treatment. Pharmacopsychiatry. 2013;46:54-58.

17. Malberg JE. Chronic antidepressant treatment increases neurogenesis in adult rat hippocampus. $J$ Neurosci. 2000;20:9104-9110.

18. Nibuya M. Chronic antidepressant administration increases the expression of cAMP response element binding protein (CREB) in rat hippocampus. J Neurosci. 1996;16:2365-2372.

19. American Psychiatric Association. Diagnostic and Statistical Manual of Mental Disorders: DSM-IV-TR. Arlington, VA, USA: American Psychiatric Publishing, Inc.; 2000.

20. Moroney JT, Bagiella E, Desmond DW, et al. Meta-analysis of the Hachinski Ischemic Score in pathologically verified dementias. Neurology. 1997;49:1096-1105.

21. Folstein MF, Folstein SE, McHugh PR. "Mini-mental state". A practical method for grading the cognitive state of patients for the clinician. J Psychiatr Res. 1975;12:189-198.

22. Manos PJ, Wu R. The Ten Point Clock test: a quick screen and grading method for cognitive impairment in medical and surgical patients. Int J Psychiatry Med. 1994;24:229-244.

23. Wechsler D. Wechsler Memory Scale (WMS-III). San Antonio, TX, USA: Psychological Corporation; 1997.

24. Benton A, Hamsher K, Varney N, Spreen O. Contribution to Neuropsychological Assessment: New York, NY, USA: Oxford University Press; 1983.

25. Karege F, Perret G, Bondolfi G, et al. Decreased serum brain-derived neurotrophic factor levels in major depressed patients. Psychiatry Res. 2002;109:143-148.

26. Chang YC, Tzeng SF, Yu L, et al. Early-life fluoxetine exposure reduced functional deficits after hypoxic-ischemia brain injury in rat pups. Neurobiol Dis. 2006;24:101-113.

27. Mowla A, Mosavinasab M, Pani A. Does fluoxetine have any effect on the cognition of patients with mild cognitive impairment? A doubleblind, placebo-controlled, clinical trial. J Clin Psychopharmacol. 2007;27:67-70

28. Zittel S, Weiller C, Liepert J. Citalopram improves dexterity in chronic stroke patients. Neurorehabil Neural Repair. 2008;22: 311-314.

29. Schmitt JA, Wingen M, Ramaekers JG, et al. Serotonin and human cognitive performance. Curr Pharm Des. 2006;12:2473-2486.

30. Sambeth A, Riedel WJ, Tillie DE, et al. Memory impairments in humans after acute tryptophan depletion using a novel gelatin-based protein drink. J Psychopharmacol. 2009;23:56-64.

31. Feeney DM, De Smet AM, Rai S. Noradrenergic modulation of hemiplegia: facilitation and maintenance of recovery. Restor Neurol Neurosci. 2004;22:175-190.

32. Maya Vetencourt JF, Sale A, Viegi A, et al. The antidepressant fluoxetine restores plasticity in the adult visual cortex. Science. 2008;320: 385-388.

33. Peng Q, Masuda N, Jiang M, et al. The antidepressant sertraline improves the phenotype, promotes neurogenesis and increases BDNF levels in the R6/2 Huntington's disease mouse model. Exp Neurol. 2008;210:154-163.

34. Geldmacher DS, Waldman AJ, Doty L, et al. Fluoxetine in dementia of the Alzheimer's type: prominent adverse effects and failure to improve cognition. J Clin Psychiatry. 1994;55:161.

35. Chen B, Dowlatshahi D, MacQueen GM, et al. Increased hippocampal BDNF immunoreactivity in subjects treated with antidepressant medication. Biol Psychiatry. 2001;50:260-265.

36. Laske C, Stransky E, Leyhe T, et al. Decreased brain-derived neurotrophic factor (BDNF)- and beta-thromboglobulin (beta-TG)- blood levels in Alzheimer's disease. Thromb Haemost. 2006;96:102-103.
37. Pliego-Rivero FB, Bayatti N, Giannakoulopoulos X, et al. Brain-derived neurotrophic factor in human platelets. Biochem Pharmacol. 1997;54: 207-209.

38. Yamamoto H, Gurney ME. Human platelets contain brain-derived neurotrophic factor. J Neurosci. 1990;10:3469-3478.

39. Radka SF, Holst PA, Fritsche M, et al. Presence of brain-derived neurotrophic factor in brain and human and rat but not mouse serum detected by a sensitive and specific immunoassay. Brain Res. 1996;709: 122-301.

40. Fujimura H, Altar CA, Chen R, et al. Brain-derived neurotrophic factor is stored in human platelets and released by agonist stimulation. Thromb Haemost. 2002;87:728-734.

41. Nakahashi T, Fujimura H, Altar CA, et al. Vascular endothelial cells synthesize and secrete brain-derived neurotrophic factor. FEBS Lett. 2000;470:113-117.

42. Donovan MJ, Miranda RC, Kraemer R, et al. Neurotrophin and neurotrophin receptors in vascular smooth muscle cells. Regulation of expression in response to injury. Am J Pathol. 1995;147:309-324.

43. Braun A, Lommatzsch M, Mannsfeldt A, et al. Cellular sources of enhanced brain-derived neurotrophic factor production in a mouse model of allergic inflammation. Am J Respir Cell Mol Biol. 1999;21: 537-546.

44. Kerschensteiner M, Gallmeier E, Behrens L, et al. Activated human $\mathrm{T}$ cells, B cells, and monocytes produce brain-derived neurotrophic factor in vitro and in inflammatory brain lesions: a neuroprotective role of inflammation? J Exp Med. 1999;189:865-870.

45. Poduslo JF, Curran GL. Permeability at the blood-brain and blood-nerve barriers of the neurotrophic factors: NGF, CNTF, NT-3, BDNF. Brain Res Mol Brain Res. 1996;36:280-286.

46. Pan W. Transport of brain-derived neurotrophic factor across the bloodbrain barrier. Neuropharmacology. 1998;37:1553-1561.

47. Karege F, Schwald M, Cisse M. Postnatal developmental profile of brain-derived neurotrophic factor in rat brain and platelets. Neurosci Lett. 2002;328:261-264.

48. Thal DR, Grinberg LT, Attems J. Vascular dementia: different forms of vessel disorders contribute to the development of dementia in the elderly brain. Exp Gerontol. 2012;47:816-824.

49. Navaratna D, Guo SZ, Hayakawa K, et al. Decreased cerebrovascular brain-derived neurotrophic factor-mediated neuroprotection in the diabetic brain. Diabetes. 2011;60:1789-1796.

50. Amoureux S, Lorgis L, Sicard P, et al. Vascular BDNF expression and oxidative stress during aging and the development of chronic hypertension. Fundam Clin Pharmacol. 2012;26:227-234.

51. Rantamaki T, Hendolin P, Kankaanpaa A, et al. Pharmacologically diverse antidepressants rapidly activate brain-derived neurotrophic factor receptor TrkB and induce phospholipase-Cgamma signaling pathways in mouse brain. Neuropsychopharmacology. 2007;32:2152-2162.

52. Kozisek ME, Middlemas D, Bylund DB. The differential regulation of BDNF and TrkB levels in juvenile rats after four days of escitalopram and desipramine treatment. Neuropharmacology. 2008;54: 251-257.

53. Surget A, Saxe M, Leman S, et al. Drug-dependent requirement of hippocampal neurogenesis in a model of depression and of antidepressant reversal. Biol Psychiatry. 2008;64:293-301.

54. Li Y, Luikart BW, Birnbaum S, et al. TrkB regulates hippocampal neurogenesis and governs sensitivity to antidepressive treatment. Neuron. 2008;59:399-412.

55. Gunstad J, Benitez A, Smith J, et al. Serum brain-derived neurotrophic factor is associated with cognitive function in healthy older adults. J Geriatr Psychiatry Neurol. 2008;21:166-170.

56. Lee JG, Shin BS, You YS, et al. Decreased serum brain-derived neurotrophic factor levels in elderly korean with dementia. Psychiatry Investig. 2009;6:299-305.

57. Yasutake C, Kuroda K, Yanagawa T, et al. Serum BDNF, TNF-alpha and IL-1beta levels in dementia patients: comparison between Alzheimer's disease and vascular dementia. Eur Arch Psychiatry Clin Neurosci. 2006;256:402-406. 


\section{Publish your work in this journal}

Clinical Interventions in Aging is an international, peer-reviewed journal focusing on evidence-based reports on the value or lack thereof of treatments intended to prevent or delay the onset of maladaptive correlates of aging in human beings. This journal is indexed on PubMed Central, MedLine, the American Chemical Society's 'Chemical Abstracts

\section{Dovepress}

Service' (CAS), Scopus and the Elsevier Bibliographic databases. The manuscript management system is completely online and includes a very quick and fair peer-review system, which is all easy to use. Visit $\mathrm{http}: / /$ www.dovepress.com/testimonials.php to read real quotes from published authors.

Submit your manuscript here: http://www.dovepress.com/clinical-interventions-in-aging-journal 